\title{
Au(I) Catalyzed HF Transfer: Tandem Alkyne Hydrofluorination and Perfluoroarene Functionalisation
}

\author{
Daniel Mulryan, Jack Rodwell, Nicholas A. Phillips, and Mark R. Crimmin* \\ Molecular Sciences Research Hub, Imperial College London, 82 Wood Lane, Shepherds Bush, London, W12 \\ $\mathrm{OBZ}, \mathrm{UK}$
}

\begin{abstract}
HF transfer reactions between organic substrates are an incredibly rare class of transformation. Such reactions require the development of new catalytic systems that can promote both defluorination and fluorination steps in a single reaction sequence. Herein, we report a novel catalytic protocol in which an equivalent of HF is generated from a perfluoroarene / nucleophile pair and transferred directly to an alkyne. The reaction is catalysed by $\left[\mathrm{Au}(\operatorname{IPr}) \mathrm{N}^{\prime} \mathrm{Pr}_{2}\right]\left(\operatorname{IPr}=N_{,} N^{\prime}-1,3-B i s(2,6-\right.$ diisopropylphenyl)imidazol-2-ylidene) and is $100 \%$ atom efficient. HF transfer generates two useful products in the form of functionalised fluoroarenes and fluoroalkenes. Mechanistic studies (rate laws, KIES, DFT calculations, competition experiments) are consistent with the Au(I) catalyst facilitating a catalytic network involving both concerted $S_{N} A r$ and hydrofluorination steps. The nature of the nucleophile impacts the turnover-limiting step. The $c S_{N} A r$ step is turnover-limiting for phenol-based nucleophiles while proteodeauration likely becomes turnover-limiting for aniline-based nucleophiles. The new approach removes the need for direct handling of HF reagents in hydrofluorination and offers new possibilities to manipulate the fluorine content of organic molecules through catalysis.
\end{abstract}

\section{Introduction}

Hydrofluorination is an essential method in synthesis. The addition of HF to unsaturated functional groups serves as an atom-efficient and expedient way to introduce fluorine atoms into organic molecules. ${ }^{1-10}$ Such substitutions are highly attractive for drug discovery and agrochemical sciences where the introduction of fluorine is known to block metabolic pathways, improve lipophilicity, modify pKa of adjacent sites, and improve binding through non-covalent interactions. ${ }^{11,12}$ 
Hydrofluorination methods are not without their technical challenges. HF is a corrosive gas and high concentrations can be fatal in contact with skin. ${ }^{13,14}$ Modified HF reagents, such as pyridinium poly(hydrogen fluoride) (Olah's reagent) or triethylamine tri(hydrogen fluoride) (TREAT-HF) have been widely adopted, and while not volatile like HF itself, they remain highly toxic and corrosive. ${ }^{8,15,16}$ Furthermore, these types of fluorinating agents are exclusively derived from HF produced from acidification of fluorite $\left(\mathrm{CaF}_{2}\right)$. There are concerns over the sustainability of such approaches. ${ }^{17,18}$ In the long-term, the fluorochemicals sector will need to resolve the twin issues of finite raw materials and the damage caused by release of fluorinated molecules into the environment.

In this paper, we report a new catalytic reaction that results in the transfer of an equivalent of HF between a fluoroarene | nucleophile pair and an alkyne. Transfer functionalisations are an emerging, highly efficient, and powerful class of reactions for synthesis, owing to their high atom economy and potential reversibility. ${ }^{19,20}$ Recent advances in this field have provided systems for the shuttling of $\mathrm{HX}$ (Figure 1a, $\mathrm{X}=\mathrm{CN},{ }^{21} \mathrm{Cl}, \mathrm{Br}^{22}, \mathrm{I}^{23}$ ) between organic fragments. ${ }^{24}$ Our approach allows the realisation of these methods for HF transfer and combines both defluorination and hydrofluorination steps in a single catalytic network. Both products of HF transfer are useful fluorinated synthons, resulting in a $100 \%$ atom economic process. The new method removes the need to directly handle HF (or related) reagents in hydrofluorination catalysis, improving safety concerns. It also represents an important step towards the chemical recycling of fluorinated compounds through re-use of their fluorine content. $^{25}$

There is limited precedent for reactions that transfer HF between organic substrates. In 2010, Kalow and Doyle reported the catalytic enantioselective reaction of benzoyl fluoride, 1,1,1,3,3,3-hexafluoro2-propanol and cyclohexene oxide (Figure 1b). ${ }^{26-29}$ The reaction resulted in the net addition of HF to the epoxide. Despite this remarkable result, a general approach to HF transfer for hydrofluorination remains elusive. Indeed, transition metal catalysts developed for $\mathrm{HX}$ shuttling $(\mathrm{X}=\mathrm{CN}, \mathrm{Cl}, \mathrm{Br}, \mathrm{I})^{21-23}$ are poor candidates to develop such reactivity due to the reluctance of carbon-fluorine bonds to participate in oxidative addition and reductive elimination steps at transition metal centres.

In 2006, Sadighi and co-workers reported that the gold(I) fluoride complex, [Au(SIPr)F], could catalyse the trans-selective addition of $\mathrm{HF}$ to an internal alkyne (SIPr = 1,3-bis(2,6-diisopropylphenyl)-4,5dihydroimidazol-2-ylidene). ${ }^{1}$ A gold(I) fluoride complex supported by a bis(phosphine) ligand, $\left[\mathrm{Au}\left({ }^{\mathrm{t}} \mathrm{BuXantPhos}\right) \mathrm{F}\right]$ has also been shown to be an on-cycle intermediate in the catalytic $\mathrm{S}_{\mathrm{N}} \mathrm{Ar}$ of perfluoroarenes ('BuXantPhos $=$ 9,9-dimethyl-4,5-bis(di-tertbutylphosphino)xanthene). ${ }^{30}$ Both 
pathways achieve catalytic turnover due to the low fluorophilicity of $\mathrm{Au}(\mathrm{I})$. These two key results suggest that $\mathrm{Au}(\mathrm{I})$ catalysts may be viable candidates for developing transfer catalysis with $\mathrm{HF}$, but only if both types of reactivity can be established within a single catalytical network, ideally by a single catalyst.

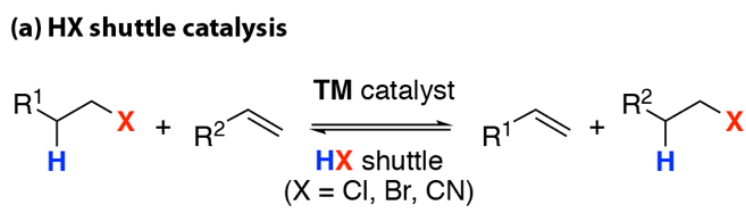

(b) HF transfer catalysis

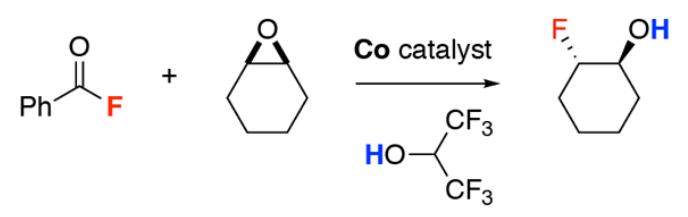

(c) this work

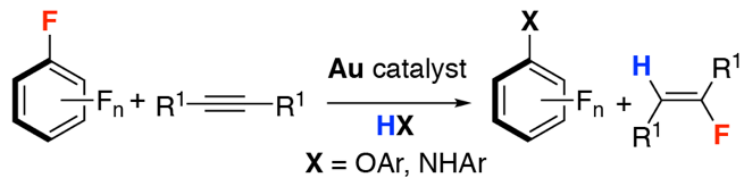

Figure 1. (a) The general reaction scheme for transition metal catalysed $\mathrm{HX}$ shuttling $(\mathrm{X}=\mathrm{Cl}, \mathrm{Br}, \mathrm{CN})$. (b) $\mathrm{HF}$ transfer catalysis (including this work).

\section{$\underline{\text { Results and Discussion }}$}

Catalyst Development: Following a campaign of catalyst screening and reaction optimisation, a new catalytic HF transfer reaction was developed (see supporting information for further details). The reaction of pentafluoropyridine, 4-methoxyphenol and diphenylacetylene in toluene at $120{ }^{\circ} \mathrm{C}$ was catalysed by $10 \mathrm{~mol} \%$ [Au(IPr)NiPr$]_{2}^{31}$ and led to the formation of corresponding biaryl ether (1a) and fluoroalkene (2a) in $>80 \%$ spectroscopic yield. This protocol transfers an equivalent of HF from the fluoroarene | nucleophile pair to the alkyne. Hydrofluorination of the alkyne shows complete selectivity for the trans-isomer. ${ }^{1,8}$ The pre-catalyst is operationally simple. While a related $\mathrm{Au}(\mathrm{I})$ amide has been applied in hydrofluorination catalysis, ${ }^{3}$ to the best of our knowledge, these types of species are limited to stoichiometric applications in fluoroarene functionalisation. ${ }^{32}$

Optimisation of the conditions highlighted the need for the reaction to be performed in a poly(tetrafluoroethylene) lined vessel to exclude the side reactions with borosilicate glassware. The limiting reagent of the reaction is the fluoroarene. This finding exemplifies the difference with traditional hydrofluorination reactions of alkynes, in which an excess of HF-reagent is typical. ${ }^{1,9,33}$ 
Here, an excess of alkyne and a slight excess of nucleophile was required for a high yield of fluoroalkene product. Control reactions in the absence of the catalyst show no HF transfer to the alkyne. Furthermore, a background reaction between 4-methoxyphenol and pentafluoropyridine showed no reaction after $16 \mathrm{~h}$ at $120^{\circ} \mathrm{C}$. These controls demonstrate that the $\mathrm{Au}(\mathrm{I})$ catalyst plays a role in both the hydrofluorination and $\mathrm{S}_{N} \mathrm{Ar}$ steps.

Reaction Scope: A range of substituted phenols and anilines were shown to be effective nucleophiles for the reaction with pentafluoropyridine and diphenylacetylene to form 1a-m (Figure 2). High to modest yields were observed with both electron-rich and electron-deficient nucleophiles, however a general decrease in the relative yield of fluoroalkene product (2a) was observed with anilines compared to phenols. The scope in fluoroarene was investigated with 4-methoxyphenol as the nucleophile and diphenylacetylene as the HF acceptor to form mixtures of $\mathbf{1 n - p}$ and $\mathbf{2 a}$. Lower yields were observed with less electron-deficient fluoroarenes and the scope is currently limited to systems known to be susceptible to $S_{N} A r$. Di-substitution products $10^{\prime}$ and $1 p^{\prime}$ ' were observed with 2,3,4,5,6pentafluorobenzonitrile and 2,3,4,5,6-pentafluoronitrobenzene allowing $>1$ equiv. of $\mathrm{HF}$ to be liberated per fluoroarene, thereby increasing the yield of the HF transfer product $2 \mathrm{a}$. Multiple $\mathrm{S}_{\mathrm{N}} \mathrm{Ar}$ reactions are expected and are consistent with previous reports for these fluoroarenes. ${ }^{34,35}$ The reaction could also be applied to both alkyl and aryl internal alkynes allowing the formation of $\mathbf{2} \mathbf{b}$ - $\mathbf{d}$ as products of HF transfer. In every case, the hydrofluorination was $100 \%$ trans-selective. ${ }^{1,8}$ Prior examples of $\mathrm{Au}(\mathrm{I})$ catalysed fluoroarene functionalisation are limited to the use of silylated nucleophiles due to the need to create a thermodynamic sink for the liberated fluoride. ${ }^{30,32,36}$ Hence, HF transfer catalysis allows expansion of the substrate scope to more convenient and synthetically accessible nucleophiles.

Both fluoroalkene and fluoroarene products have synthetic utility. Substituted fluoroarenes are applied in liquid crystal displays, ${ }^{37,38}$ light-emitting diodes ${ }^{39,40}$ and as precursors for fluorinated synthons. ${ }^{41,42}$ 1a has also been highlighted as a protected form of phenol, and can regenerate the phenol and pentafluoropyridine under mild conditions. ${ }^{43}$ Vinyl fluoride functional groups, such as

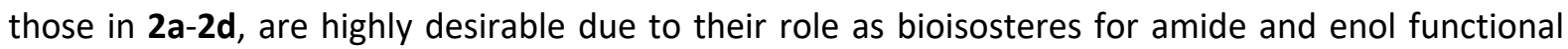
groups. ${ }^{11,44,45}$ 

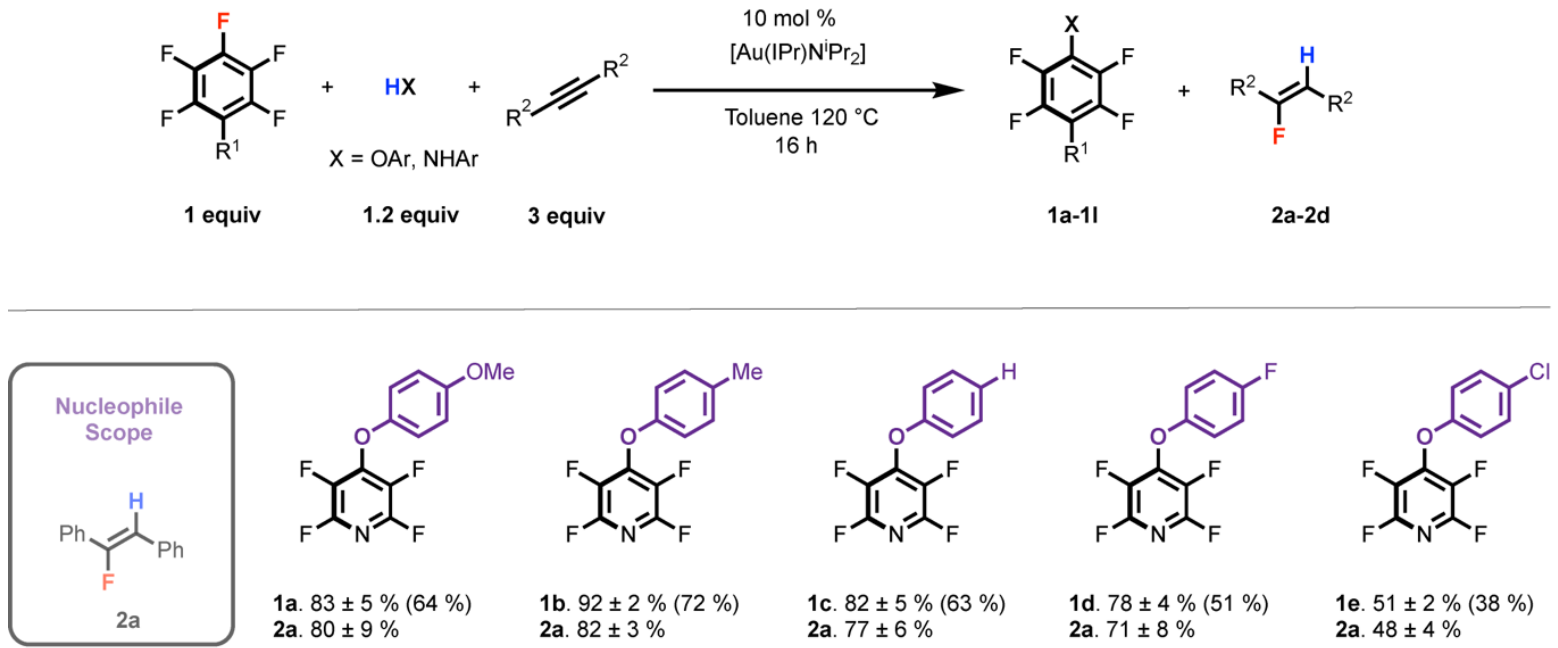

1b. $92 \pm 2 \%(72 \%)$

1c. $82 \pm 5 \%(63 \%)$

1d. $78 \pm 4 \%(51 \%)$

1e. $51 \pm 2 \%$ (38\%) 2a. $80 \pm 9 \%$

2a. $82 \pm 3 \%$<smiles>N#Cc1ccc(Oc2c(F)c(F)nc(F)c2F)cc1</smiles><smiles>CN(C)c1ccc(Nc2c(F)c(F)nc(F)c2F)cc1</smiles><smiles>COc1ccc(Nc2c(F)c(F)nc(F)c2F)cc1</smiles><smiles>Cc1ccc(Nc2c(F)c(F)nc(F)c2F)cc1</smiles><smiles>Fc1nc(F)c(F)c(Nc2ccccc2)c1F</smiles><smiles>Fc1ccc(Nc2c(F)c(F)nc(F)c2F)cc1</smiles>

1f. $49 \pm 1 \%(32 \%)$

1g. $96 \pm 3 \%(76 \%)$

1h. $96 \pm 7 \%$ (72\%)

1i. $93 \pm 4 \%(81 \%)$

1j. $74 \pm 2 \%$ (68\%)

2a. $34 \pm 7 \%$

1k. $81 \pm 2 \%(53 \%)$ 2a. $48 \pm 8 \%$

2a. $32 \pm 6 \%$<smiles>Fc1nc(F)c(F)c(Nc2ccc(Cl)cc2)c1F</smiles><smiles>N#Cc1ccc(Nc2c(F)c(F)nc(F)c2F)cc1</smiles>

Fluoroarene Scope

1m. $64 \pm 6 \%$ (47 \%)

11. $87 \pm 4 \%(69 \%)$ 2a. $41 \pm 6 \%$<smiles>COc1ccc(Oc2c(F)c(F)c(C(F)(F)F)c(F)c2F)cc1</smiles>

1n. $55 \pm 8 \%$ (34\%) 2a. $40 \pm 5 \%$<smiles>COc1ccc(Oc2c(F)c(F)c(F)c([N+]#N)c2F)cc1</smiles>

10. $70 \pm 1 \%$ (58\%) 2a. $97 \pm 3 \%$

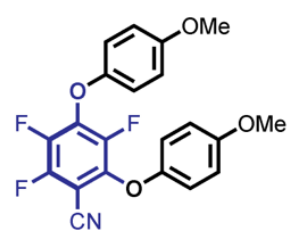

1o'. $13 \pm 1 \%$ (7 \%)<smiles>COc1ccc(Oc2c(F)c(F)c(F)c(F)c2F)cc1</smiles>

$\mathrm{NO}_{2}$

1p. $63 \pm 4 \%(51 \%)$

2a. $110 \pm 7 \%$<smiles>COc1ccc(Oc2c(F)c(F)c(Oc3ccc(OC)cc3)c([N+](=O)[O-])c2F)cc1</smiles>

1p'. $18 \pm 1 \%(9 \%)$

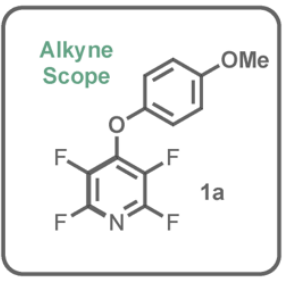

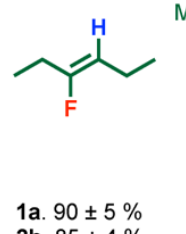

2b. $85 \pm 4 \%$<smiles>COc1ccc(/C=C(\F)c2ccc(/C=C(\F)c3ccc(F)cc3)cc2)cc1</smiles>

1a. $91 \pm 4 \%(76 \%)$

1a. $86 \pm 5 \%(68 \%)$ 2c. $89 \pm 3 \%(62 \%)$ 2d. $82 \pm 4 \%(57 \%)$

Figure 2. HF transfer reaction scope catalysed by $\left[\mathrm{Au}(\mathrm{IPr}) \mathrm{N}^{\mathrm{P}} \mathrm{Pr}_{2}\right]$. Reactions were performed with $0.1: 1: 1.2: 3$ equivalents of catalyst : fluoroarene $(0.04 \mathrm{M})$ : nucleophile : alkyne. Yields of fluoroarene (1a-1p) and fluoroalkene (2a-2d) were calculated from ${ }^{19} \mathrm{~F}$ NMR spectroscopy, using a fluorobenzene internal standard. Isolated yields were obtained from scale-up reactions and are shown in parenthesis. Isolated yields of $2 \mathrm{a}$ are not reported due to this compound co-eluting with diphenylacetylene. Reactions were performed in triplicate and standard deviations are reported with a $99 \%$ confidence level. 
Kinetic Analysis: Kinetic analysis was used to gain insight into the new catalytic protocol. The experimentally determined empirical rate law for the reaction of 4-methoxyphenol (HX), pentafluoropyridine (fluoroarene) and excess diphenylacetylene with $10 \mathrm{~mol} \%$ [Au(IPr)Ni $\left.\mathrm{Pr}_{2}\right]$ (cat) is given in eq. 1.

$$
\text { Rate }(\text { phenol })=k^{\prime}[\mathrm{HX}][\text { fluoroarene }][\text { cat }]
$$

The reaction was found to be $1^{\text {st }}$ order in $\mathrm{HX}, 1^{\text {st }}$ order in fluoroarene and $1^{\text {st }}$ order in catalyst. Initial rates were used to determine the catalyst order, while pseudo first-order conditions and time course data over 3 half-lives were used to determine the order in $\mathrm{HX}$ and fluoroarene. ${ }^{46}$ Due to the strict requirement to run the reaction in an excess of alkyne, ( $>2$ equivalents, outlined by previous studies ${ }^{1}$ and confirmed in optimisation reactions) orders for this reagent were not determined.

Kinetic experiments were also run using 4-methoxyaniline as a nucleophile in place of 4methoxyphenol. The interpretation of this data is complicated by the observation that $\mathbf{1 h}$ and $\mathbf{2 a}$ form at different rates for this nucleophile (vide infra). Despite this limitation, the analysis suggests the formation of $2 \mathrm{a}$ is $0^{\text {th }}$ order in fluoroarene and $1^{\text {st }}$ order in $\mathrm{HX}$, and strongly indicate that the mechanism may change depending on the nature of the nucleophile. This finding is further supported by the measurement of KIEs which indicate a small isotope of $1.1 \pm 0.1$ effect for the reaction of 4methoxyphenol / $d_{1}-4$-methoxyphenol but a clear primary KIE of $2.8 \pm 0.3$ for the formation of $2 a$ from 4-methoxyaniline / $d_{2}$-4-methoxyaniline. These KIE values are consistent across both independent rates measurements and intermolecular competition experiments (Figure 3).
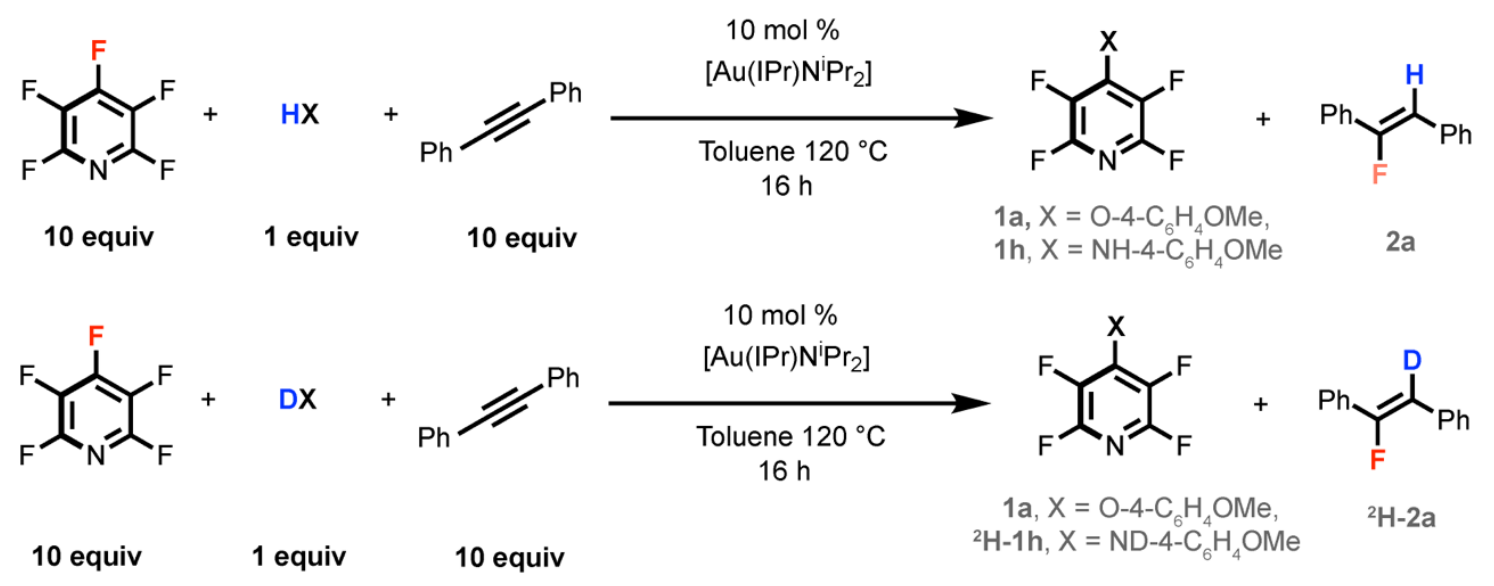

$\operatorname{KIE}($ phenol $)=1.1 \pm 0.1$

$\mathrm{KIE}($ aniline $)=\mathbf{2 . 8 \pm 0 . 3}$

Figure 3. KIEs determined by independent rates. 
DFT Calculations: DFT calculations were performed to supplement the kinetic data and used as a foundation with which to build a mechanistic model. The PBEO functional, which has previously been used to model $\mathrm{Au}(\mathrm{l})$ interactions with alkynes, ${ }^{47,48}$ and $6-31 \mathrm{G}^{* *}$ basis set were used for all atoms other than $\mathrm{Au}$, for which the SDDAll pseudo potential was applied. A single-point empirical dispersion correction (D3) with Becke-Johnson damping and solvent correction (PCM, $\varepsilon=2.38$ ) was applied to the energies of all stationary points. Pathways were calculated for both the reaction of 4-methoxyphenol and 4-methoxyaniline with pentafluoropyridine and diphenylacetylene (Figure 4). Initially, a simple model constructed from a catalytic cycle without considering off-cycle species was considered. Toste, Bergman, and coworkers have demonstrated that $\left[\mathrm{Au}(\mathrm{IPr}) \mathrm{N}^{\mathrm{i} P r_{2}}\right]$ does not react with diphenylacetylene below $75{ }^{\circ} \mathrm{C}$, but that this species is capable of deprotonating weak acids (e.g. fluorene $\mathrm{pKa}=23$ in THF). ${ }^{31}$ Pre-catalyst initiation by protonolysis with $\mathrm{HX}$ was considered to be facile and $\left[\mathrm{Au}(\mathrm{IPr}) \mathrm{N}^{\mathrm{i} P r_{2}}\right]$ assumed to react with 4-methoxyphenol $(\mathrm{pKa}=19)^{49}$ and 4-methoxyaniline (pKa $30)^{50}$ to form $[\mathrm{Au}(\mathrm{IPr}) \mathrm{X}]\left(\mathrm{X}=\mathrm{O}-4-\mathrm{C}_{6} \mathrm{H}_{4} \mathrm{OMe}, \mathrm{NH}-4-\mathrm{C}_{6} \mathrm{H}_{4} \mathrm{OMe}\right)$.

The reaction of $[\mathrm{Au}(\mathrm{IPr}) \mathrm{X}]\left(\mathrm{X}=\mathrm{O}-4-\mathrm{C}_{6} \mathrm{H}_{4} \mathrm{OMe}\right)$ with pentafluoropyridine is calculated to occur by a concerted $\mathrm{S}_{N} A$ r mechanism by TS-1 $\left(\Delta \mathrm{G}^{\ddagger}=+21.8 \mathrm{kcal} \mathrm{mol}^{-1}\right)$ to form 1a alongside [Au(IPr)F]. TS-1 bears all the features expected for a concerted $S_{N} A r\left(c S_{N} A r\right)$ process with charge accumulation and pyramidalization occurring at the ipso-carbon of the electrophile. The $\{\mathrm{Au}(\mathrm{IPr})\}^{+}$fragment interacts with both the alkoxide nucleophile and the fluoride leaving group in TS-1, and concerted bond breaking and formation was confirmed by IRC calculations which show only a single TS connecting starting materials and products. Prior computational studies on Au-catalysed hydrodefluorination of fluoroarenes have modelled redox pathways but consider trigonal planar three-coordinate $\mathrm{Au}(\mathrm{I})$ intermediates supported by bis(phosphine) ligands rather than two-coordinate linear geometries. ${ }^{36}$

The hydrofluorination sequence evolves from $[\mathrm{Au}(\mathrm{IPr}) \mathrm{F}]$. Explicit solvation of this species with the nucleophile was considered, leading to a series of structures stabilised by F---H-X hydrogen bonding interactions. Upon addition of diphenylacetylene, fluoride dissociation from Int-1 to form Int-2 is calculated to be endergonic $\left(\Delta \mathrm{G}_{\mathrm{rxn}}^{\circ}=+6.3 \mathrm{kcal} \mathrm{mol}^{-1}\right)$ and occurs by a facile interchange mechanism by TS-2 $\left(\Delta \mathrm{G}^{\ddagger}=+7.9 \mathrm{kcal} \mathrm{mol}^{-1}\right) \cdot \pi$-Complexation of the alkyne to the cationic $\mathrm{Au}(\mathrm{I})$ fragment in Int-2 is supported by NBO calculations; second order perturbation analysis reveals both $\sigma$-donation and $\pi$ backdonation components to the bonding (see supporting information). This bonding interaction renders the alkyne ligand of Int-2 susceptible to nucleophilic attack by the fluoride ion. TS-3 shows that slippage of the alkyne from an $\eta^{2}$ towards an $\eta^{1}$ bonding mode occurs during this reaction 
pathway as evidenced by the asymmetry of the Au-C bond distances ( 2.13 vs $2.44 \AA$ ). ${ }^{51}$ Fluorination by TS-3 ultimately leads to the vinyl $\mathrm{Au}(\mathrm{I})$ species Int-3 with complete stereochemical control. The energy span between Int-1 and TS-3 defines the barrier for the fluorination sequence $\left(\Delta G^{\ddagger}=+19.4\right.$ $\mathrm{kcal}^{\mathrm{mol}}{ }^{-1}$ ) which is lower than the barrier for the ${ }^{2} S_{N} A r$ step. Finally, protodeauration of Int-3 by $\mathrm{HX}$ occurs via TS-4 $\left(\Delta \mathrm{G}^{\ddagger}=+16.4 \mathrm{kcal} \mathrm{mol}^{-1}\right)$ and leads to the hydrofluorination product $2 \mathrm{a}$ while regenerating the active catalyst $[\mathrm{Au}(\mathrm{IPr}) \mathrm{X}]$. NBO analysis reveals partial $\mathrm{C}---\mathrm{H}$ bond formation and $\mathrm{C}---$ Au bond breaking in the transition state, while IRC calculations are consistent with association of the resulting charged fragments $\{\mathrm{Au}(\mathrm{IPr})\}^{+}$and $\mathrm{X}^{-}$occurring afterwards. ${ }^{52-56}$ The overall transformation is calculated to be exergonic $\left(\Delta \mathrm{G}_{\mathrm{rxn}}^{\circ}=-23.1 \mathrm{kcal} \mathrm{mol}^{-1}\right)$ with the $\mathrm{S}_{N} A r$ step being turnover limiting.
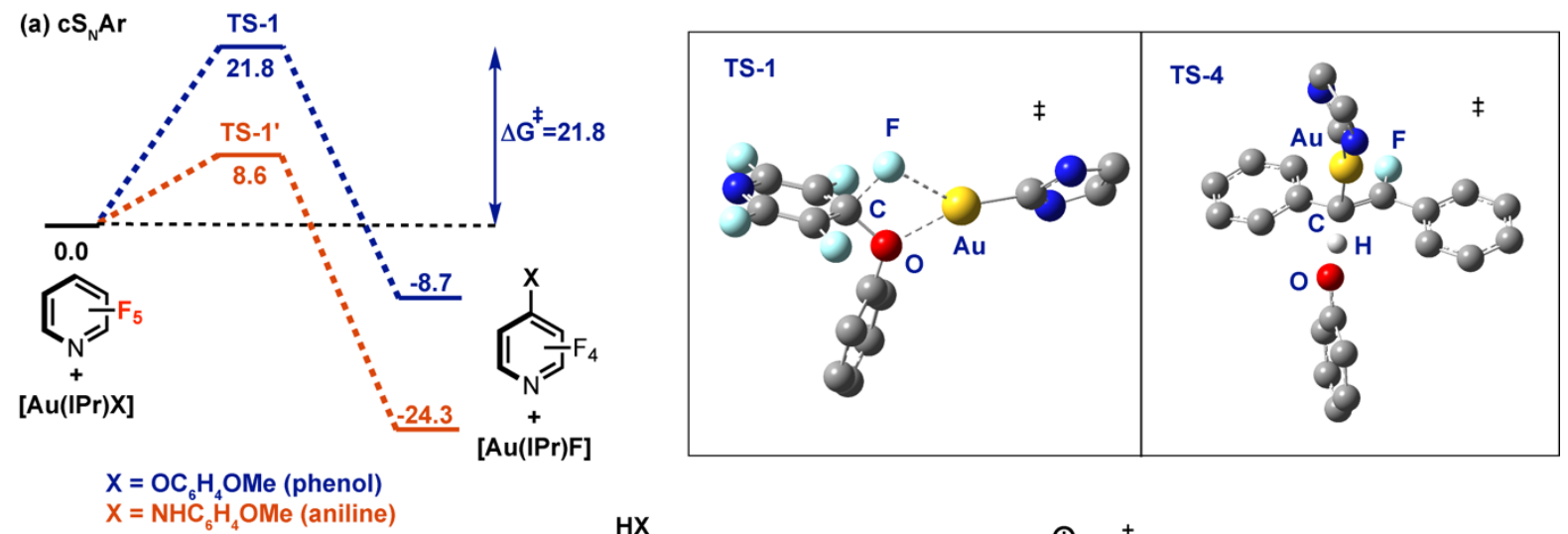

(b) hydrofluorination

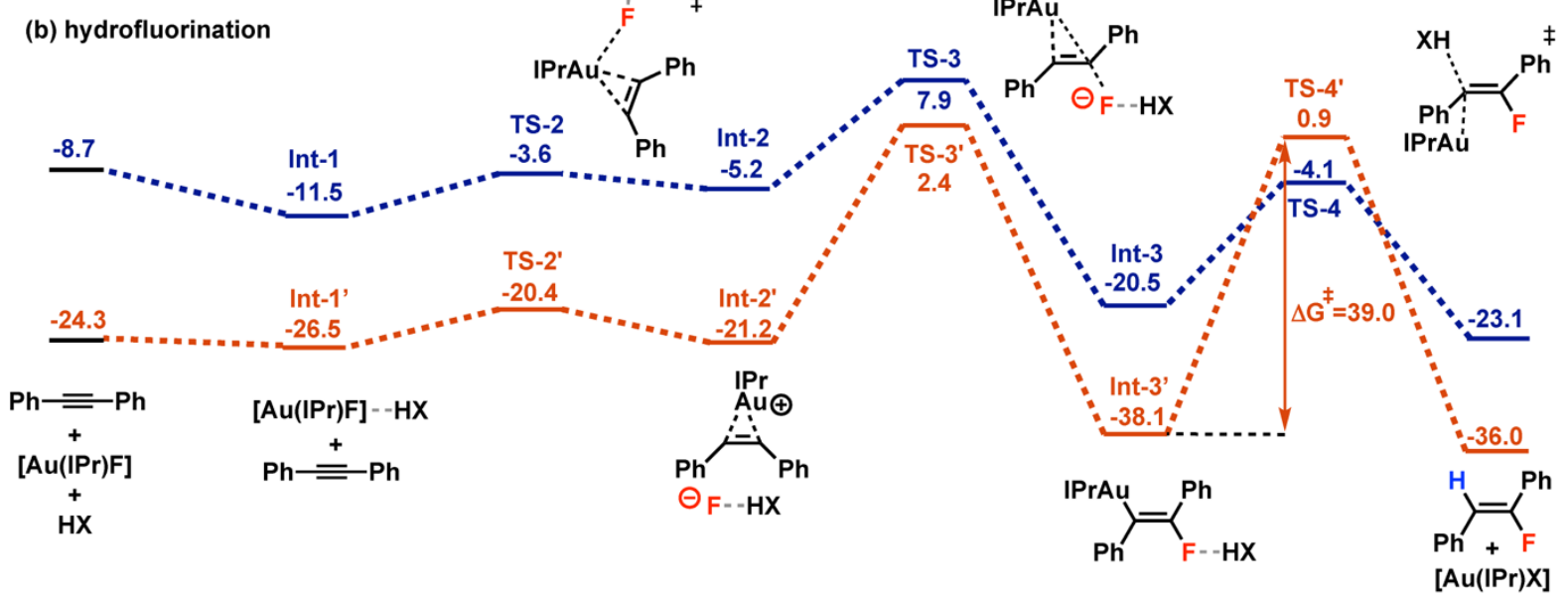

Figure 4. DFT calculated pathways for (a) $C S_{N} A r$ and (b) hydrofluorination reaction pathways. (inset) models of TS-1 and TS-4 showing geometries of key steps.

A closely related pathway was calculated for the reaction of $[\mathrm{Au}(\operatorname{IPr}) \mathrm{X}]\left(\mathrm{X}=\mathrm{NH}-4-\mathrm{C}_{6} \mathrm{H}_{4} \mathrm{OMe}\right)$ with pentafluoropyridine and diphenylacetylene albeit with significantly modified barriers for each of the steps. The improved nucleophilicity of the amide ligand of $\left[\mathrm{Au}(\mathrm{IPr}) \mathrm{NH}-4-\mathrm{C}_{6} \mathrm{H}_{4} \mathrm{OMe}\right](\mathrm{NPA}$ charges $\mathrm{Au}=$ $+0.42, \mathrm{~N}=-1.00$ ) over the alkoxide of [Au(IPr)O-4- $\mathrm{C}_{6} \mathrm{H}_{4} \mathrm{OMe}$ (NPA charges $\mathrm{Au}=+0.45, \mathrm{O}=-0.82$ ), 
alongside the reduced pKa of $\mathrm{HO}-4-\mathrm{C}_{6} \mathrm{H}_{4} \mathrm{OMe}$ compared to $\mathrm{H}_{2} \mathrm{~N}-4-\mathrm{C}_{6} \mathrm{H}_{4} \mathrm{OMe}$ impacts key transition state barriers. The $S_{N} A r$ step by TS-1' is now a low energy process $\left(\Delta G^{\ddagger}=+8.6 \mathrm{kcal} \mathrm{mol}^{-1}\right)$ and as such is no longer predicted to be the turnover-limiting step. In contrast, the protodeauration step from the $\mathrm{Au}(\mathrm{I})$ vinyl intermediate Int-3' to TS-4' involves a large energy barrier $\left(\Delta \mathrm{G}^{\ddagger}=+39.0 \mathrm{kcal} \mathrm{mol}^{-1}\right)$ and is not only predicted to be the slowest step of the catalytic sequence, but the activation energy is also large enough to question if it is accessible under the reaction conditions $\left(120^{\circ} \mathrm{C}, 16 \mathrm{~h}\right)$.

Comparison of the TS-1 and TS-1' allows identification of the key features that led to the lowering of this barrier for the $\mathrm{CS}_{N} A r$ step. The $\mathrm{C}---\mathrm{X}$ and $\mathrm{C}---\mathrm{F}$ distances in TS-1 are $1.44(\mathrm{X}=0)$ and $1.65 \AA$ respectively, while the $\mathrm{X}-\mathrm{C}-\mathrm{F}$ angle is $89.9^{\circ}$. In contrast, TS-1' possesses a more open structure with a much longer $\mathrm{C}---\mathrm{X}$ interaction of $1.92 \AA(X=N)$, shorter $\mathrm{C}-\mathrm{F}$ distance of $1.36 \AA$ and obtuse $\mathrm{X}-\mathrm{C}-\mathrm{F}$ angle of $94.1^{\circ}$. These metrics suggest that C-F bond breaking in TS-1' is less advanced than in TS-1. The accumulation of charge on key moieties in these transition states is consistent with this argument as TS-1 ( $\left.\Delta \mathrm{q}: A u=+0.07, O=+0.20, \mathrm{C}_{\text {ipso }}=+0.04, \mathrm{~F}=-0.23\right)$ involves greater charge separation than TS$1^{\prime}\left(\Delta q: A u=+0.03, N=+0.12, C_{\text {ipso }}=+0.03, F=-0.13\right) .{ }^{57}$ A competition experiment in which excess 4methoxyphenol and 4-methoxyaniline were reacted with pentafluoropyridine and 10 mol\% [Au(IPr)NiPr$\left.r_{2}\right]$ led exclusively to $1 \mathbf{h}$ in preference to $1 \mathbf{a}$. This finding reflects the large energy difference between TS-1' and TS-1 $\left(\Delta \Delta \mathrm{G}^{\ddagger}=13.2 \mathrm{kcal} \mathrm{mol}^{-1}\right)$.

Further comparisons can be made between the protodeauration transition states TS-4 and TS-4'. Protodeauration involves direct breaking of the $\mathrm{H}-\mathrm{X}$ bond $(\mathrm{X}=\mathrm{O}, \mathrm{N})$ through deprotonation by the Au-C moiety. ${ }^{52-56}$ In TS-4 the C--- $-\mathrm{-}--\mathrm{X}$ bond angle is $174.1^{\circ}$ while the $\mathrm{H}-\mathrm{C}-\mathrm{Au}$ angle is $90.7^{\circ}$ and this reflects the orthogonality between the reacting ligand and Au centre. The protodeauration transition states TS-4 and TS-4' both involve isomerisation of the vinyl ligand from a $\sigma$ - to $\pi$-coordination mode. This reorganisation leads to an elongation of the $\mathrm{Au}-\mathrm{C}$ bond length (e.g. Int-3, 2.04; TS-4, 2.18) and charge accumulation on the $\mathrm{C}$ atom adjacent to $\mathrm{Au}$ (e.g. Int-3, -0.44; TS-4, -0.57) both of which facilitate protonation. The acidity of the $\mathrm{H}-\mathrm{X}$ moiety is a key factor in determining the activation barrier for this step. The transition state geometries would be expected to be consistent with a primary KIE if this step becomes turnover limiting.

In combination, the empirical rate-laws, the KIEs, and the DFT calculations are fully consistent with a change of turnover-limiting step depending on the nucleophile. For, 4-methoxyphenol the ${ } S_{N} A r$ is 
expected to be turnover-limiting with a small or no KIE. For 4-methoxyaniline the proteodeauration step becomes turnover-limiting; the reaction rate no longer depends on [fluoroarene], and a primary KIE is expected. Given the apparent sensitivity of these key steps to the electronics of the nucleophile, further DFT calculations were undertaken in which the nucleophile was modified through variation of the substituent at the 4-position (see supporting information for details). These calculations revealed clear free energy relationships between the transition state barriers and Hammett parameters $\left(\sigma_{\mathrm{p}}\right)$ of the nucleophile. In no case did varying the electronics of phenol or aniline change the predicted turnover-limiting step for each of these systems; the switch in mechanism requires a complete switch in nucleophile rather than perturbation of its electronic structure.

A Catalytic Network: Taken in combination, these data suggest a complex catalytic cycle involving both $\mathrm{CS}_{N} \mathrm{Ar}$ and hydrofluorination mechanisms within a single reaction network. Further insight into the difference behaviour of the two nucleophile types was obtained by following the concentration of key species (including HF) over the complete time-course of these reactions (Figure 5). In the case of pentafluoropyridine, 4-methoxyphenol and diphenylacetylene, formation of products 1a and 2a was concurrent and no HF build up was observed. For the same reaction with 4-methoxyaniline, formation of product $\mathbf{1 h}$ occurred independently of formation of $\mathbf{2 a}$. HF was identified as a reaction intermediate by a broad and concentration dependent resonance in the ${ }^{19} \mathrm{~F}$ NMR spectrum at $\delta=150.5-152.5 \mathrm{ppm}$. Given the reaction conditions, it remains likely that the HF interacts with 4-methoxyaniline in solution (through proton transfer or hydrogen-bonding) ${ }^{58}$. An induction period was observed for the formation of $\mathbf{2 a}$ and an increase in the rate of formation of this species is observed as the HF concentration increases. 
a)

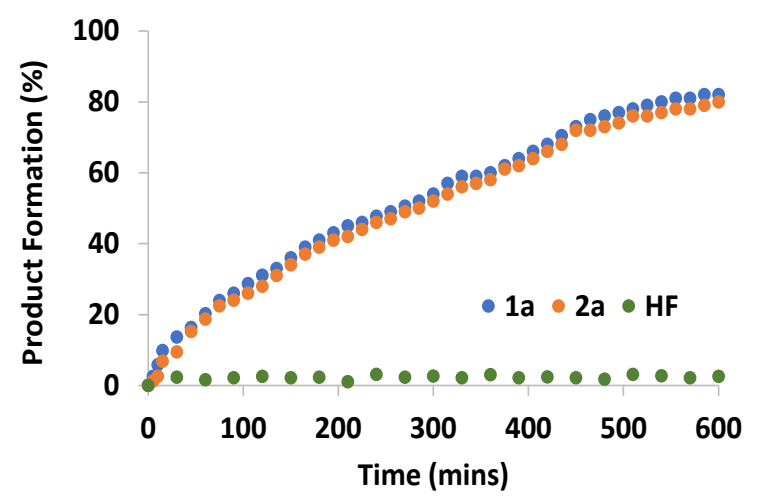

b)

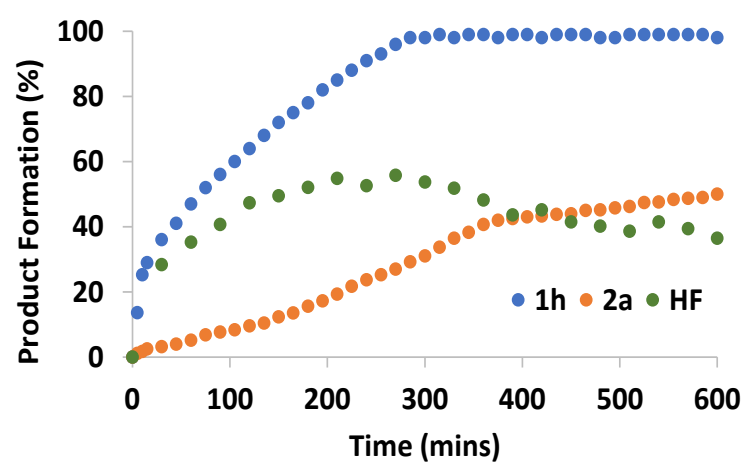

Figure 5. Plots for concentration of fluoroarene and fluoroalkene products, and HF intermediate over time for the reaction of pentafluoropyridine and diphenylacetylene with (a) 4-methoxyphenol and (b) 4-methoxyaniline.

The catalytic network in Figure 5 is a plausible reaction mechanism. This network explains the combined data and calculations. The reaction potentially operates in two different regimes depending on the nature of the nucleophile. In regime 1 , for phenol-based nucleophiles, the ${ }^{2} S_{N} A r$ step is expected to be slow and turnover-limiting. As such, any $[\mathrm{Au}(\mathrm{IPr}) \mathrm{F}]$ generated is expected to be consumed in the onwards hydrofluorination sequence. While $[\mathrm{Au}(\mathrm{IPr}) \mathrm{F}]$ can also potentially react with $\mathrm{HX}$ to liberate $\mathrm{HF}$ and regenerate the active catalyst, this reaction is calculated to be endergonic $(\mathrm{X}=$ O-4- $\mathrm{C}_{6} \mathrm{H}_{4} \mathrm{OMe} ; \Delta \mathrm{G}^{\circ}=12.4 \mathrm{kcal} \mathrm{mol}^{-1}, \Delta \mathrm{G}^{\ddagger}=14.0 \mathrm{kcal} \mathrm{mol}^{-1}$ ) and should be reversible under the catalytic conditions. Protodeauration with $\mathrm{HX}$ is facile, leading to synchronous formation of the products $\mathbf{1}$ and 2 and no build-up of HF during the reaction.

In regime 2, for aniline based nucleophiles, the ${ } S_{N} A r$ step is now fast and the hydrofluorination sequence is slow and likely turnover limiting. The extremely high calculated activation barrier for proteodeauration with $\mathrm{HX}\left(\Delta \mathrm{G}^{\ddagger}=39.0 \mathrm{kcal} \mathrm{mol}^{-1}\right)$ suggests that this step may only be a minor 
contributor under the reaction conditions. Instead, reaction of $[\mathrm{Au}(\mathrm{IPr}) \mathrm{F}]$ with $\mathrm{HX}$ could produce a bypass in the catalytic cycle, resulting in generation of HF. While again calculated to be endergonic and reversible $\left(X=\mathrm{NH}-4-\mathrm{C}_{6} \mathrm{H}_{4} \mathrm{OMe} ; \Delta \mathrm{G}^{\circ}=12.4 \mathrm{kcal} \mathrm{mol}^{-1}, \Delta \mathrm{G}^{\ddagger}=13.5 \mathrm{kcal} \mathrm{mol}^{-1}\right)$, if this bypass step operated in combination with the consumption of HF in the proteodeauration step it may prove thermodynamically viable. If combined with the fast $\mathrm{CS}_{N} \mathrm{Ar}$ step, this bypass mechanism would lead to the asynchronous production of $\mathbf{1}$ and $\mathbf{2}$ along with the potential build-up of HF as a reaction intermediate. Control reactions revealed that under catalytic conditions $\mathbf{1 h}$ forms in near quantitative yield, alongside $\mathrm{HF}$, even if diphenylacetylene was omitted from the reaction mixture. As $\mathrm{HF}$ is susceptible to off-cycle side reactions, this mechanism would explain the lower yield of $\mathbf{2}$ for anilinebased nucleophiles compared to phenol-based nucleophiles (Figure 2).

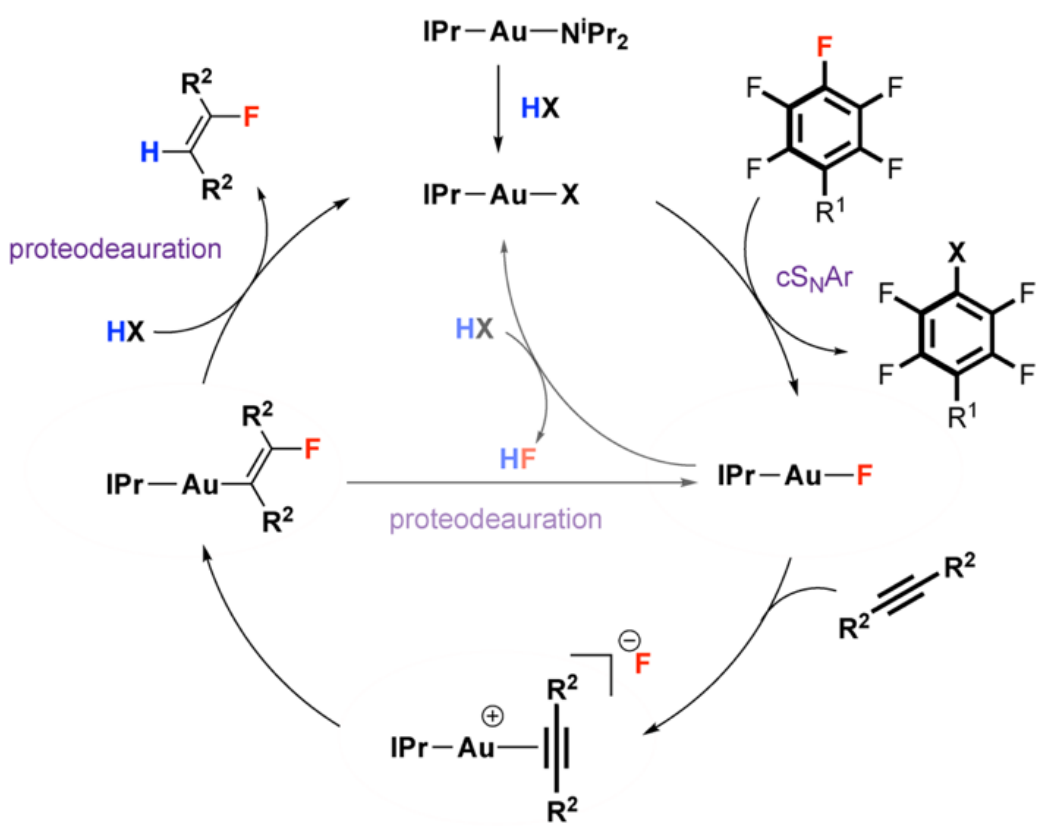

Figure 6. Plausible catalytic network for HF transfer.

This mechanistic hypothesis could be used to improve the yields of the HF transfer product $\mathbf{2}$ when carrying out reactions with aniline-based nucleophiles. The catalytic reaction to form $1 \mathrm{~h}$ in $83 \pm 5 \%$ yield generates $50 \pm 2 \%$ of $\mathbf{2 a}$ as a co-product (Figure 2 ). When the standard conditions are repeated but 1.2 equiv. of 4-methoxyphenol is added to the reaction mixture, $1 \mathrm{~h}$ is still formed as the exclusive ${ }_{C S} \operatorname{Ar}$ product in $92 \pm 5 \%$ but the yield of $2 a$ improves to $72 \pm 3 \%$. The finding is consistent with the addition of the phenol limiting bypass catalysis by accelerating the protodeauration step. 


\section{Conclusions}

In summary, a novel $\mathrm{Au}(\mathrm{I})$-catalysed $\mathrm{HF}$ transfer reaction for the tandem hydrofluorination of alkynes and functionalisation of fluoroarenes has been developed. HF is generated through the reaction of perfluoroarene with a nucleophile, obviating the need for direct handling of HF based reagents and providing an operationally simple approach to fluorination catalysis. Through kinetics analysis, competition experiments and DFT calculations, a detailed understanding of the catalytic network involved in HF transfer has been obtained. These studies showed that the rate of production and distribution of products is dependent on the nature of the nucleophile. This mechanistic understanding was exploited to improve the efficiency of HF transfer catalysis. In the longer-term, we believe that these results will provide a foundation for the development of new catalytic approaches to transfer fluorine containing groups between molecules and recycle fluorinated compounds.

\section{$\underline{\text { References }}$}

(1) Akana, J. A.; Bhattacharyya, K. X.; Müller, P.; Sadighi, J. P. Reversible C-F Bond Formation and the Au-Catalyzed Hydrofluorination of Alkynes. J. Am. Chem. Soc. 2007, 129 (25), 7736-7737.

(2) Gorske, B. C.; Mbofana, C. T.; Miller, S. J. Regio- and Stereoselective Synthesis of Fluoroalkenes by Directed Au(I) Catalysis. Org. Lett. 2009, 11 (19), 4318-4321.

(3) Okoromoba, O. E.; Han, J.; Hammond, G. B.; Xu, B. Designer HF-Based Fluorination Reagent: Highly Regioselective Synthesis of Fluoroalkenes and Gem-Difluoromethylene Compounds from Alkynes. J. Am. Chem. Soc. 2014, 136 (41), 14381-14384.

(4) He, G.; Qiu, S.; Huang, H.; Zhu, G.; Zhang, D.; Zhang, R.; Zhu, H. Cu(I)- or Ag(I)-Catalyzed Regioand Stereocontrolled Trans-Hydrofluorination of Ynamides. Org. Lett. 2016, 18 (8), 18561859.

(5) Zhu, G.; Qiu, S.; Xi, Y.; Ding, Y.; Zhang, D.; Zhang, R.; He, G.; Zhu, H. (IPr)CuF-Catalyzed $\alpha$-Site Regiocontrolled Trans-Hydrofluorination of Ynamides. Org. Biomol. Chem. 2016, 14 (32), 7746-7753.

(6) Gómez-Herrera, A.; Nahra, F.; Brill, M.; Nolan, S. P.; Cazin, C. S. J. Sequential Functionalization of Alkynes and Alkenes Catalyzed by Gold(I) and Palladium(II) N-Heterocyclic Carbene Complexes. ChemCatChem 2016, 8 (21), 3381-3388.

(7) Zeng, X.; Liu, S.; Hammond, G. B.; Xu, B. Divergent Regio- and Stereoselective Gold-Catalyzed Synthesis of $\alpha$-Fluorosulfones and $\beta$-Fluorovinylsulfones from Alkynylsulfones. Chem. Eur. J. 2017, 23 (49), 11977-11981.

(8) O'Connor, T. J.; Toste, F. D. Gold-Catalyzed Hydrofluorination of Electron-Deficient Alkynes: Stereoselective Synthesis of $\beta$-Fluoro Michael Acceptors. ACS Catal. 2018, 8 (7), 5947-5951.

(9) Gauthier, R.; Mamone, M.; Paquin, J.-F. Gold-Catalyzed Hydrofluorination of Internal Alkynes Using Aqueous HF. Org. Lett. 2019, 21 (22), 9024-9027.

(10) Guo, R.; Qi, X.; Xiang, H.; Geaneotes, P.; Wang, R.; Liu, P.; Wang, Y.-M. Stereodivergent Alkyne Hydrofluorination Using Protic Tetrafluoroborates as Tunable Reagents. Angew. Chem. Int. Ed. 2020, 59 (38), 16651-16660.

(11) Meanwell, N. A. Fluorine and Fluorinated Motifs in the Design and Application of Bioisosteres for Drug Design. J. Med. Chem. 2018, 61 (14), 5822-5880.

(12) Inoue, M.; Sumii, Y.; Shibata, N. Contribution of Organofluorine Compounds to Pharmaceuticals. ACS Omega 2020, 5 (19), 10633-10640. 
(13) Segal, E. B. First Aid for a Unique Acid: HF. Chem. Health Saf. 1998, 5 (5), 25-28.

(14) Segal, E. B. First Aid for a Unique Acid, HF: A Sequel. Chem. Health Saf. 2000, 7 (1), 18-23.

(15) Olah, G. A.; Welch, J. T.; Vankar, Y. D.; Nojima, M.; Kerekes, I.; Olah, J. A. Synthetic Methods and Reactions. 63. Pyridinium Poly(Hydrogen Fluoride) (30\% Pyridine-70\% Hydrogen Fluoride): A Convenient Reagent for Organic Fluorination Reactions. J. Org. Chem. 1979, 44 (22), 3872-3881.

(16) Kirk, K. L. Fluorination in Medicinal Chemistry: Methods, Strategies, and Recent Developments. Org. Process Res. Dev. 2008, 12 (2), 305-321.

(17) Harsanyi, A.; Sandford, G. Organofluorine Chemistry: Applications, Sources and Sustainability. Green Chem. 2015, 17 (4), 2081-2086.

(18) Caron, S. Where Does the Fluorine Come From? A Review on the Challenges Associated with the Synthesis of Organofluorine Compounds. Org. Process Res. Dev. 2020, 24 (4), 470-480.

(19) Bhawal, B. N.; Morandi, B. Shuttle Catalysis-New Strategies in Organic Synthesis. Chem. Eur. J. 2017, 23 (50), 12004-12013.

(20) Bhawal, B. N.; Morandi, B. Catalytic Transfer Functionalization through Shuttle Catalysis. ACS Catal. 2016, 6 (11), 7528-7535.

(21) Bhawal, B. N.; Reisenbauer, J. C.; Ehinger, C.; Morandi, B. Overcoming Selectivity Issues in Reversible Catalysis: A Transfer Hydrocyanation Exhibiting High Kinetic Control. J. Am. Chem. Soc. 2020, 142 (25), 10914-10920.

(22) Yu, P.; Bismuto, A.; Morandi, B. Iridium-Catalyzed Hydrochlorination and Hydrobromination of Alkynes by Shuttle Catalysis. Angew. Chem. Int. Ed. 2020, 59 (7), 2904-2910.

(23) Petrone, D. A.; Franzoni, I.; Ye, J.; Rodríguez, J. F.; Poblador-Bahamonde, A. I.; Lautens, M. Palladium-Catalyzed Hydrohalogenation of 1,6-Enynes: Hydrogen Halide Salts and Alkyl Halides as Convenient HX Surrogates. J. Am. Chem. Soc. 2017, 139 (9), 3546-3557.

(24) Wang, D.; Astruc, D. The Golden Age of Transfer Hydrogenation. Chem. Rev. 2015, 115 (13), 6621-6686.

(25) Cousins, I. T.; DeWitt, J. C.; Glüge, J.; Goldenman, G.; Herzke, D.; Lohmann, R.; Ng, C. A.; Scheringer, M.; Wang, Z. The High Persistence of PFAS Is Sufficient for Their Management as a Chemical Class. Environ. Sci.: Processes Impacts 2020, 22 (12), 2307-2312.

(26) Kalow, J. A.; Doyle, A. G. Enantioselective Ring Opening of Epoxides by Fluoride Anion Promoted by a Cooperative Dual-Catalyst System. J. Am. Chem. Soc. 2010, 132 (10), 3268-

(27) Kalow, J. A.; Doyle, A. G. Mechanistic Investigations of Cooperative Catalysis in the Enantioselective Fluorination of Epoxides. J. Am. Chem. Soc. 2011, 133 (40), 16001-16012.

(28) Kalow, J. A.; Doyle, A. G. Enantioselective Fluoride Ring Opening of Aziridines Enabled by Cooperative Lewis Acid Catalysis. Tetrahedron 2013, 69 (27), 5702-5709.

(29) Graham, T. J. A.; Lambert, R. F.; Ploessl, K.; Kung, H. F.; Doyle, A. G. Enantioselective Radiosynthesis of Positron Emission Tomography (PET) Tracers Containing [18F]Fluorohydrins. J. Am. Chem. Soc. 2014, 136 (14), 5291-5294.

(30) Hu, J.-Y.; Zhang, J.; Wang, G.-X.; Sun, H.-L.; Zhang, J.-L. Constructing a Catalytic Cycle for C-F to $\mathrm{C}-\mathrm{X}(\mathrm{X}=\mathrm{O}, \mathrm{S}, \mathrm{N})$ Bond Transformation Based on Gold-Mediated Ligand Nucleophilic Attack. Inorg. Chem. 2016, 55 (5), 2274-2283.

(31) Johnson, M. W.; Shevick, S. L.; Toste, F. D.; Bergman, R. G. Preparation and Reactivity of Terminal Gold(i) Amides and Phosphides. Chem. Sci. 2013, 4 (3), 1023-1027.

(32) Lv, H.; Zhan, J.-H.; Cai, Y.-B.; Yu, Y.; Wang, B.; Zhang, J.-L. $\pi-\pi$ Interaction Assisted Hydrodefluorination of Perfluoroarenes by Gold Hydride: A Case of Synergistic Effect on C-F Bond Activation. J. Am. Chem. Soc. 2012, 134 (39), 16216-16227.

(33) Nahra, F.; Patrick, S. R.; Bello, D.; Brill, M.; Obled, A.; Cordes, D. B.; Slawin, A. M. Z.; O'Hagan, D.; Nolan, S. P. Hydrofluorination of Alkynes Catalysed by Gold Bifluorides. ChemCatChem 2015, 7 (2), 240-244.

(34) Krishnan, R.; Parthiban, A. Regioselective Preparation of Functional Aryl Ethers and Esters by Stepwise Nucleophilic Aromatic Substitution Reaction. J. Fluor. Chem. 2014, 162, 17-25. 
(35) Mulryan, D.; White, A. J. P.; Crimmin, M. R. Organocatalyzed Fluoride Metathesis. Org. Lett. 2020, 22 (23), 9351-9355.

(36) Zhan, J.-H.; Lv, H.; Yu, Y.; Zhang, J.-L. Catalytic C-F Bond Activation of Perfluoroarenes by Tricoordinated Gold(I) Complexes. Adv. Synth. Catal., 2012, 354 (8), 1529-1541.

(37) Kirsch, P. Fluorine in Liquid Crystal Design for Display Applications. J. Fluor. Chem. 2015, 177, 29-36.

(38) Kirsch, P.; Bremer, M. Nematic Liquid Crystals for Active Matrix Displays: Molecular Design and Synthesis. Angew. Chem. Int. Ed. 2000, 39 (23), 4216-4235.

(39) Kamata, T.; Sasabe, H.; Watanabe, Y.; Yokoyama, D.; Katagiri, H.; Kido, J. A Series of Fluorinated Phenylpyridine-Based Electron-Transporters for Blue Phosphorescent OLEDs. J. Mater. Chem. C 2016, 4 (5), 1104-1110.

(40) Ragni, R.; Punzi, A.; Babudri, F.; Farinola, G. M. Organic and Organometallic Fluorinated Materials for Electronics and Optoelectronics: A Survey on Recent Research. Eur. J. Org. Chem. 2018, 2018 (27-28), 3500-3519.

(41) Dolbier, W. R. Fluorine Chemistry at the Millennium. J. Fluor. Chem. 2005, 126 (2), 157-163.

(42) Lv, H.; Cai, Y.-B.; Zhang, J.-L. Copper-Catalyzed Hydrodefluorination of Fluoroarenes by Copper Hydride Intermediates. Angew. Chem. Int. Ed. 2013, 52 (11), 3203-3207.

(43) Brittain, W. D. G.; Cobb, S. L. Tetrafluoropyridyl (TFP): A General Phenol Protecting Group Readily Cleaved under Mild Conditions. Org. Biomol. Chem. 2019, 17 (8), 2110-2115.

(44) Dutheuil, G.; Couve-Bonnaire, S.; Pannecoucke, X. Diastereomeric Fluoroolefins as Peptide Bond Mimics Prepared by Asymmetric Reductive Amination of $\alpha$-Fluoroenones. Angew. Chem. Int. Ed. 2007, 46 (8), 1290-1292.

(45) Burkhart, J. P.; Weintraub, P. M.; Gates, C. A.; Resvick, R. J.; Vaz, R. J.; Friedrich, D.; Angelastro, M. R.; Bey, P.; Peet, N. P. Novel Steroidal Vinyl Fluorides as Inhibitors of Steroid C17(20) Lyase. bioorg. Med. Chem. 2002, 10 (4), 929-934.

(46) As 1a and 2a form at identical rates in these reactions, orders for the entire process can be confidently assigned by monitoring either starting material consumption or formation of $1 \mathrm{a}$ or 2 a.

(47) Kang, R.; Chen, H.; Shaik, S.; Yao, J. Assessment of Theoretical Methods for Complexes of Gold(I) and Gold(III) with Unsaturated Aliphatic Hydrocarbon: Which Density Functional Should We Choose? J. Chem. Theory Comput. 2011, 7 (12), 4002-4011.

(48) Li, H.; Liu, J.; Abosede A., O.; Bao, X. Computational Insights into the Mechanisms of Au(i)Catalysed Intramolecular Addition of the Hydroxylamine Group onto Alkynes. Org. Chem. Front. 2017, 4 (6), 1130-1136.

(49) Bordwell, F. G.; McCallum, R. J.; Olmstead, W. N. Acidities and Hydrogen Bonding of Phenols in Dimethyl Sulfoxide. J. Org. Chem. 1984, 49 (8), 1424-1427.

(50) Bordwell, F. G.; Algrim, D. J. Acidities of Anilines in Dimethyl Sulfoxide Solution. J. Am. Chem. Soc. 1988, 110 (9), 2964-2968.

(51) Eisenstein, O.; Hoffmann, R. Transition-Metal Complexed Olefins: How Their Reactivity toward a Nucleophile Relates to Their Electronic Structure. J. Am. Chem. Soc. 1981, 103 (15), 4308-4320.

(52) Biasiolo, L.; Trinchillo, M.; Belanzoni, P.; Belpassi, L.; Busico, V.; Ciancaleoni, G.; D’Amora, A.; Macchioni, A.; Tarantelli, F.; Zuccaccia, D. Unexpected Anion Effect in the Alkoxylation of Alkynes Catalyzed by N-Heterocyclic Carbene (NHC) Cationic Gold Complexes. Chem. Eur. J. 2014, 20 (45), 14594-14598.

(53) Ciancaleoni, G.; Belpassi, L.; Zuccaccia, D.; Tarantelli, F.; Belanzoni, P. Counterion Effect in the Reaction Mechanism of NHC Gold(I)-Catalyzed Alkoxylation of Alkynes: Computational Insight into Experiment. ACS Catal. 2015, 5 (2), 803-814.

(54) Trinchillo, M.; Belanzoni, P.; Belpassi, L.; Biasiolo, L.; Busico, V.; D’Amora, A.; D’Amore, L.; Del Zotto, A.; Tarantelli, F.; Tuzi, A.; Zuccaccia, D. Extensive Experimental and Computational 
Study of Counterion Effect in the Reaction Mechanism of NHC-Gold(I)-Catalyzed Alkoxylation of Alkynes. Organometallics 2016, 35 (5), 641-654.

(55) D’Amore, L.; Ciancaleoni, G.; Belpassi, L.; Tarantelli, F.; Zuccaccia, D.; Belanzoni, P. Unraveling the Anion/Ligand Interplay in the Reaction Mechanism of Gold(I)-Catalyzed Alkoxylation of Alkynes. Organometallics 2017, 36 (12), 2364-2376.

(56) Lu, Z.; Li, T.; Mudshinge, S. R.; Xu, B.; Hammond, G. B. Optimization of Catalysts and Conditions in Gold(I) Catalysis-Counterion and Additive Effects. Chem. Rev. 2021, 121 (14), 8452-8477.

(57) $\Delta q$ Is defined here as the change in NPA charge on an atom, on transitioning from $[\mathrm{Au}(\mathrm{IPr}) \mathrm{X}]+$ pentafluoropyridine to TS-1 or TS-1'.

(58) Szatyłowicz, H.; Krygowski, T. M.; Panek, J. J.; Jezierska, A. H-Bonded Complexes of Aniline with HF/F- and Anilide with HF in Terms of Symmetry-Adapted Perturbation, Atoms in Molecules, and Natural Bond Orbitals Theories. J. Phys. Chem. A 2008, 112 (40), 9895-9905.

TOC:
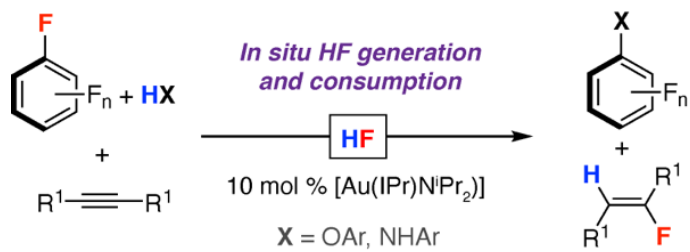